\title{
Quantitative Assessment of Canalicular Bile Formation in Isolated Hepatocyte Couplets Using Microscopic Optical Planimetry
}

\author{
A. Gautam, O. C. Ng, M. Strazzabosco, and J. L. Boyer \\ Department of Medicine and Liver Center, Yale University School of Medicine, New Haven, Connecticut 06510
}

\begin{abstract}
Isolated rat hepatocyte couplets (IRHC) are primary units of bile secretion that accumulate fluid in an enclosed canalicular space with time in culture. We have quantitated the rate of canalicular secretion in IRHC cultured for 4-8 h by measuring the change in canalicular space volume by video-microscopic optical planimetry using high resolution Nomarski optics. Electron microscopic morphometric studies revealed significant increases in canalicular membrane area after 4-6 h in culture. Canalicular secretion in basal L-15 medium (3.8 \pm 1.3 $\mathrm{fl} / \mathrm{min}$ ) increased significantly with the choleretic bile salts $(10 \mu \mathrm{M})$, taurocholate, and ursodeoxycholate $(14 \pm 7 \mathrm{fl} / \mathrm{min}$ each). Secretion rates after exposure to bile acids correlated directly with the canalicular surface area before stimulation. In contrast, expansion times after stimulation varied inversely with initial canalicular volumes. Ursodeoxycholic acid failed to produce a hypercholeresis at 10-, 100-, or 200- $\mu \mathrm{M}$ concentrations compared with taurocholate, either in normal or taurinedepleted IRHC.

The present findings establish that rates of canalicular bile secretion can be quantitated in IRHC by serial optical planimetry, both in the basal state and after stimulation with bile acids. Furthermore, ursodeoxycholate does not acutely induce hypercholeresis at the canalicular level in this model. Rather, both taurocholic and ursodeoxycholic acids induced secretion in proportion to the surface area of the canalicular membrane. The IRHC are a useful model to identify canalicular choleretics and for studies of canalicular bile formation.
\end{abstract}

\section{Introduction}

Hepatocytes are polarized cells that form bile by secreting solutes and water across an apical membrane into small canalicular spaces that exist between adjacent hepatocytes. This primary secretion may be further modified by reabsorption or secretion by the bile ductular epithelium at distal sites.

Although significant progress has been achieved in understanding the mechanisms of canalicular bile secretion (1-5),

Portions of this work were presented in preliminary form at the Annual Meetings of the American Gastroenterology Association, 1986 and 1987 (1986. Gastroenterology. 90:1727, and 1987. Gastroenterology. 92:1734).

Please address all correspondence to Dr. Anil Gautam, Liver Unit, 1080 LMP, Yale University School of Medicine, New Haven, CT 06510.

Received for publication 8 September 1987 and in revised form 20 September 1988.

J. Clin. Invest.

(c) The American Society for Clinical Investigation, Inc.

0021-9738/89/02/0565/09 \$2.00

Volume 83, February 1989, 565-573 knowledge is still incomplete, in part because the small size $(1-2 \mu \mathrm{m})$ and inaccessibility of the bile canaliculus in the intact liver precludes direct quantitative measurements of the primary secretion. Most studies of canalicular bile secretion have relied, therefore, on indirect methods including isolated perfused liver systems, where bile is sampled from biliary cannulas at distal sites after contact with and possible modification by bile ductules and ducts. Isolated membrane subfractions obtained from specific hepatocyte membrane domains have also been useful in localizing membrane transport systems and determining solute driving forces (6-8). However, liver cells are disrupted in this in vitro system and the findings may not always mirror the physiology in vivo. Thus, uncertainties still exist about the mechanisms of primary canalicular secretory processes, as summarized in several reviews (3-5). This report describes a new way to directly quantitate canalicular secretory events in a cell culture system.

Isolated rat hepatocyte couplets (IRHC) ${ }^{1}$ permit assessment of canalicular secretory events in an isolated cell culture system that eliminates confounding effects of blood flow, hormonal influences, or transport events in the bile duct system $(9,10)$. IRHC consist of two nondissociated hepatocytes enclosing a sealed canalicular space. When the couplets are placed in short-term culture, secretion accumulates within the space resulting in progressive expansion followed by spontaneous collapse or contractions, as originally observed by Oshio and Phillips (11).

Several studies have demonstrated that IRHC display many characteristics of a functionally intact primary unit of canalicular bile formation $(9,11-14)$. Studies of the structural characteristics of the IRHC $(10,15,16)$ indicate that freshly isolated hepatocyte pairs reorganize their apical membranes to the retained canalicular pole that is demarcated by tight junction complexes between the two hepatocytes. Thus, the IRHC retains its structural secretory polarity. A detailed electrophysiologic analysis of this primary canalicular secretory unit has recently been completed (12).

In the present report we describe a method to quantitate the volume of the canalicular space by microscopic optical planimetry using differential interference contrast (DIC) video-microscopy (17-19). By determining the change in volume of the canalicular space in response to choleretic stimuli, we have quantitated rates of canalicular bile formation in this isolated hepatocyte couplet system (20). In addition, we have compared the canalicular choleretic effects of the bile salts, sodium taurocholate and ursodeoxycholate.

Ursodeoxycholate in high concentrations has been reported to produce a unique hypercholeresis of bicarbonateenriched bile where bile flow is significantly greater than that

1. Abbreviations used in this paper: DIC, differential interference contrast; IRHC, isolated rat hepatocyte couplets; L-15, Liebowitz-15 medium. 
accounted for by the biliary bile acid output $(21,22)$, and choleresis correlates both with biliary $\mathrm{HCO}_{3}^{-}$concentration and output and the amount of unconjugated ursodeoxycholate in bile. Both hypercholeresis and $\mathrm{HCO}_{3}^{-}$output are blocked by the $\mathrm{Na}^{+}-\mathrm{H}^{+}$inhibitor, amiloride, and its more potent and presumably more specific analogues $(22,23)$. The mechanisms and sites of action of ursodeoxycholate remain undetermined but may include: $(a)$ stimulation of bile formation at the level of the hepatocyte (24) by stimulation of the basolateral $\mathrm{Na}^{+} / \mathrm{H}^{+}$exchanger (25) leading to passive or active transport of $\mathrm{HCO}_{3}^{-}$into the canalicular space; $(b)$ primary stimulation of other unknown ion pumps by large intracellular accumulations of unconjugated bile acid; $(c)$ cholehepatic shunting, where the unconjugated bile acid is excreted into bile at the canalicular level but is protonated and partly reabsorbed passively distally in the biliary tree, thereby recycling across the canalicular membrane to affect an apparent hypercholeresis relative to the total amount of bile acid finally excreted in bile (26); and $(d)$ stimulation of ion transporters distally at the level of the bile ductules or ducts.

In this study we determine whether ursodeoxycholate in various concentrations produces a hypercholeretic effect in isolated hepatocyte couplets compared with similar concentrations of taurocholate.

\section{Methods}

Isolation of hepatocyte couplets. IRHC were prepared in the Hepatocyte Isolation Core laboratory of the Liver Center from fed, male Sprague-Dawley rats (180-250 g; Camm Research Lab Animals, Wayne, $\mathrm{NJ})$ as previously described $(9,27)$ by a modification of the nonrecirculating collagenase perfusion method of Seglen. New modifications were the inclusion of $0.8 \mathrm{U}$ of trypsin inhibitor (Sigma Chemical Co., St. Louis, MO)/U of tryptic activity in the collagenase and 1 $\mathrm{mg}$ of the clostripan inhibitor, $N$-tosyl L-lysyl chloromethane hydrochloride, in the collagenase buffer to minimize nonspecific proteolytic activity of these contaminants. The resulting cell preparations containing $0.8-2.2 \times 10^{7}$ hepatocytes $/ g$ of liver were $>95 \%$ hepatocytes, and $91.7 \pm 2.9 \%$ of the usable preparations excluded trypan blue ( $n$ $=231$ isolations) and were relatively enriched in hepatocyte couplets ( $31.6 \pm 8.6 \%$ of cells were IRHC in 138 consecutive isolations). Preparations in which $<85 \%$ cells excluded trypan blue were discarded. The final cell suspension was plated onto glass coverslip fragments in 35 $\times 10$-mm plastic tissue culture dishes at a cell concentration of 0.75 $\times 10^{5} / \mathrm{ml}$ of Liebowitz-15 medium (L-15; Gibco Laboratories, Grand Island, NY) containing $50 \mathrm{U}$ of penicillin and $50 \mu \mathrm{g}$ of strepto$\operatorname{mycin} / \mathrm{ml}$.

Measurement of canalicular volume and quantitative assessment of canalicular bile formation in IRHC. The unique structural and secretory properties of the hepatocyte couplet were exploited to quantitate the rate of primary canalicular secretion both in a basal medium and after stimulation with choleretic bile salts. The approach used was $(a)$ to quantitate the canalicular space volume accurately by obtaining cross-sectional dimensions at several known intervals through the entire canalicular depth by the technique of microscopic optical planimetry using DIC video-microscopy $(17-20)$, and $(b)$ to calculate the rate of canalicular secretion from the rate of change of the canalicular volume with time as secretion was elaborated.

Optical and video systems for optical planimetry. Canalicular volume was measured by microscopic optical planimetry, originally described by Spring et al. $(17,18)$. The principle of this method is shown in Fig. 1. Using DIC, or Nomarski optics, which has a very shallow depth of focus (0.3-0.4 $\mu \mathrm{m}$ under our optical conditions), cross-sectional images of the canaliculus were obtained at multiple optical planes through the depth of the canaliculus at $1-\mu \mathrm{m}$ intervals. On the right is a three-dimensional projection of the canalicular space gener-

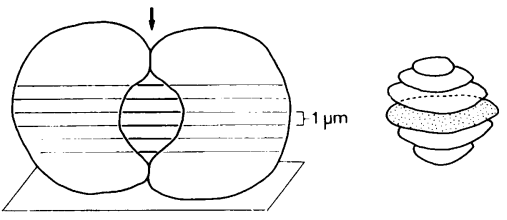

Figure 1. Principle of optical planimetry. A couplet adherent to a coverslip fragment is viewed from the side. Using Nomarski optics (see text for detailed specifications), multiple optical sections are obtained $1 \mu \mathrm{m}$ apart through the depth of the canaliculus. A computer-generated three-dimensional projection is shown on the right. Each section is quantitated for cross-sectional area and perimeter using an image analyzer. The sum of measured cross-sectional areas $\times 1 \mu \mathrm{m}=$ canalicular volume; the sum of measured perimeters $\times 1 \mu \mathrm{m}=$ canalicular surface area.

ated by a video-plan II image analyzer (Carl Zeiss, Inc., Thornwood, NY). The extremely shallow depth of focus of Nomarski optics permits spacing the steps $0.5-1 \mu \mathrm{m}$ apart so that each optical plane is true without bleeding of images of optical planes above and below into the plane of focus. At each step the perimeter and the cross-sectional surface area of the canalicular space were quantitated. The sum of the measured cross-sectional areas $\times$ displacement $(1 \mu \mathrm{m}$ in this case) = canalicular volume (in femtoliters), and the sum of the measured perimeters $\times 1 \mu \mathrm{m}=$ canalicular surface area (in square micrometers). In preliminary studies a comparison of canalicular area and volume measurements was made using $0.5-, 1.0-$, and $2.0-\mu \mathrm{m}$ steps. No signifcant differences were noted in the coefficient of variation between 0.5 and $2.0-\mu \mathrm{m}$ steps in the measured parameters. Steps of 1.0 or $2.0 \mu \mathrm{m}$ were used thereafter.

Optical planimetry was carried out in the following manner. IRHC were cultured for $\sim 4 \mathrm{~h}$ in L-15 medium at $37^{\circ} \mathrm{C}$ on $5 \times 5-\mathrm{mm}$ glass coverslip fragments, which were then transferred for observation into a 1.2-mm-thick flow-through lucite chamber in which perfusing buffer was kept at $37 \pm 0.1^{\circ} \mathrm{C}$ by a feedback-controlled heating device. A "lung" of gas-permeable tubing was used to aerate the buffer with $100 \% \mathrm{O}_{2}$ or $95 \% \mathrm{O}_{2} / 5 \% \mathrm{CO}_{2}$. The perfusion chamber was placed on the stage of an inverted microscope (IM35; Carl Zeiss, Inc.) and couplets were studied temporally with DIC optics at a magnification of 4,660 using a $63 \times$ oil objective (Plan Neoflaur with a numerical aperture of 1.25; Carl Zeiss, Inc.) with matching DIC Wollaston prisms and an aplanatic-achromatic condenser with a 1.4 pol front (oil immersion) lens. The iris diaphragm could be opened fully with video-microscopy for the highest resolution. Contrast was adjusted by selecting the appropriate black level for the video camera circuit using a gain and black control device. Using this optical system a lateral resolution of $0.3 \mu \mathrm{m}$ and vertical resolution of $0.35 \mu \mathrm{m}$ was realized throughout. The lens combination used to achieve this resolution limits the condenser-objective lens working distance to $\sim 1 \mathrm{~mm}$. Chambers of lucite, with a bottom composed of a $100-\mu$ m-thick coverslip, were used with a similar coverslip used on top of the chamber to which the oil immersion condenser was applied. A thin flow-through chamber was thus established in which buffer exchanges occurred within $10 \mathrm{~s}$ at a flow rate of $1 \mathrm{ml} / \mathrm{min}$. Koehler illumination was set daily. Corrections for the differences in the refractive indices of the immersion oil, glass coverslip fragments, and cells were made by calibrating the image-analysis system with a stage micrometer kept in the plane of the examined cells.

The couplets being studied could then be either photographed directly or imaged at high resolution using a video camera (67; DageMTI Inc., Wabash, MI). Images were stored on a 9240XD Panasonic high resolution $3 / 4$ " video cassette recorder $(480 \times 480$ line resolution $)$. Analyses could subsequently be performed on the stored data by tracing the canalicular perimeter at each step with a cursor directly from the video overlay using a digitizing tablet and a videoplan II image analyzer (Carl Zeiss, Inc.).

For each volume quantitation the top pole of the canaliculus was located and the fine focus of the microscope advanced at $1-\mu \mathrm{m}$ inter- 
vals through the depth of the canaliculus. With a 2-3-s exposure at each step the entire record of 8-10 cuts per canaliculus was obtained within $30 \mathrm{~s}$. Volume quantitations were repeated at 3-5-min intervals in the untreated basal state to identify IRHC with expanding canalicular spaces since these are most suitable for quantitating rates of canalicular expansion in response to choleretics. Others were not used for experiments. From these measurements the basal secretory rates in unmodified L-15 medium were quantitated. Observations were repeated at 3-5-min intervals after exposure to bile acids to obtain stimulated secretory rates. This technique of quantitation of volumes of cells and interspaces by microscopic optical planimetry readily permitted accurate detection of volume changes that were at least $5 \%$ greater than preceding or baseline measurements (28). Approximately half of the IRHC with expanded canaliculi were unsuitable for study since they were already in the process of collapsing or contracting. These canaliculi may respond by reexpansion at variable time periods after exposure to choleretic bile acids with expansions presumably occurring when the canalicular limiting membranes reseal.

To study the effect of various concentrations of ursodeoxycholate on the bile flow rate in couplets, IRHC were maintained in either L-15 or in MEM supplemented with $10 \%$ FCS. Before volume measurements the coverslip fragments were either transferred from L- 15 into $\mathrm{KRB}, \mathrm{pH} 7.4$, and equilibrated for $10 \mathrm{~min}$ before measurements of bile flow rates in this bicarbonate-containing perfusate, or transferred from MEM to a MEM perfusion medium to continue a bicarbonate environment. Additional experiments were conducted on IRHC pretreated for $2 \mathrm{~d}$ with $\beta$-alanine ( $20 \mathrm{mmol} / \mathrm{kg}$ body wt daily for $2 \mathrm{~d}$ instilled into the stomach by gavage) to deplete them of taurine according to the method of Shaffer and Kocsis (29) as used by Kitani and Kanai (24). Couplets isolated from $\beta$-alanine-treated rats were further maintained in culture media supplemented with $\beta$-alanine $(0.1 \mathrm{mg} / 3 \mathrm{ml}$ medium $)$ for 3-6 $\mathrm{h}$ before study. This treatment to deplete IRHC of taurine was designed to minimize taurine conjugation of ursodeoxycholate to tauroursodeoxycholate which is not hypercholeretic in rats. Taurinedepleted rats demonstrate an earlier and more vigorous hypercholeresis in response to ursodeoxycholic acid administration (24), presumably since taurine conjugation is minimized from the beginning of the experiment rather than resulting from a progressive depletion of endogenous taurine stores in the normal liver after prolonged exposure to large doses of ursodeoxycholic acid.

Electron microscopic morphometry of the canalicular membrane. The canalicular membranes in IRHC are lined by normal-appearing (0.1-0.2- $\mu \mathrm{m}$-thick) microvilli immediately after isolation and after 4-8 $\mathrm{h}$ in culture. Morphometric studies were carried out on thin-section electron micrographs of isolated couplets in culture to $(a)$ determine the percentage of canalicular space volume occupied by these microvilli, since measurements by optical planimetry do not take into account the canalicular microvilli that are below the level of resolution by light microscopy, and $(b)$ to assess if there was a change in the number of microvilli per unit length of canalicular membrane or in the percentage of cross-sectional area occupied by microvilli with time in culture.

Two groups of IRHC were studied. Freshly isolated hepatocytes were plated onto glass coverslip fragments in L-15 medium for 10-15 min to allow the couplets to adhere to the glass surface and then processed for electron microscopic study. This group is referred to as the $0-15$-min sample. Couplets were also cultured on the coverslip in L-15 medium at $37^{\circ} \mathrm{C}$ for $4-8$ (mean 6 ) h. All cultured adherent cells were fixed with $1.5 \%$ glutaraldehyde in $0.1 \mathrm{M}$ cacodylate buffer, $\mathrm{pH}$ 7.4, postfixed with $1 \%$ osmium tetroxide in $0.1 \mathrm{M}$ cacodylate buffer containing $1 \%$ potassium ferrocyanide, en bloc stained with $0.5 \%$ uranyl acetate in $0.15 \mathrm{M}$ sodium chloride, dehydrated in graded alcohol, and embedded in epon. The coverslips were mounted on the top of the flat end of the BEEM capsule (Ted Pella, Inc., Tustin, CA). After polymerization the coverslips were cracked and the cells were sectioned horizontal to the culture plane. Thin sections were stained with uranyl acetate and lead citrate and examined with an electron microscope (10B; Carl Zeiss, Inc.) at $80 \mathrm{kV}$. Morphometric analyses were

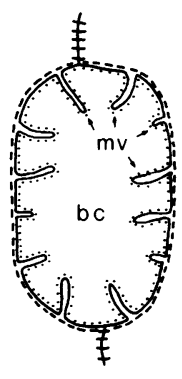

Figure 2. Diagrammatic representation of quantitative morphometric analyses of electron micrograph cross-sections of canalicular spaces in IRHC. The image analysis cursor was traced first over the entire surface, including microvilli and canalicular perimeter $(\cdots \cdots)($ measurement $A)$; the second tracing was over the canalicular perimeter across the base of the microvilli (-- -) (measurement B). $b c$, canalicular lumen; $m v$, microvilli.

then carried out on electron micrographs of canalicular cross-sections (final magnification 12,740) using a Video-Plan II image analyzer (Carl Zeiss, Inc.) as illustrated in Fig. 2.

The image analysis cursor was first traced over the entire perimeter including each microvillus (A) to yield the actual canalicular crosssectional surface area and total membrane perimeter. The cursor was then traced over the perimeter of the cross-section across the base of the microvilli (B), thus excluding them from calculations. The difference $(A-B)$ reflects the amount of membrane accounted for by the microvilli. Analysis (B) corresponds more closely to the volume measurements carried out by light microscopy during optical planimetry from the video overlay, since visualization of microvilli is not possible at this resolution.

Statistical methods. The significance of the change between the bile acid stimulated secretory rate relative to basal secretory rate in the same couplet was determined using the paired $t$ test. Stimulated secretory rates between choleretic agents were compared using the unpaired $t$ test.

\section{Results}

IRHC cultured on glass without serum remained spherical for 3-8 $\mathrm{h}$ after isolation as previously reported. The membranes limiting the canalicular space resealed after isolation and the space then expanded as secretion accumulated. Dilated canalicular spaces were detectable by Nomarski optics by $90-120$ min after initial plating and, as previously described, $12.7 \pm 4.7 \%$ of IRHC displayed canalicular spaces with diameter $>3 \mu \mathrm{m}$ after $4 \mathrm{~h}$ in culture (10). After $4 \mathrm{~h}$ the ultrastructure of the couplets was usually normal except for an increase in autophagic vacuoles, and the canalicular membrane displayed microvilli on its surface.

Since the volume of the canalicular space, as measured by optical planimetry in the hepatocyte couplet system, expanded with time over the 4-h period in culture after initial plating, it was important to determine if this expansion represented accrual of new apical membrane as opposed to distension of the original canalicular lumen. Since the latter was lined with microvilli after isolation of the couplets, it was possible that the lumen might dilate as pressure built up within this closed space, with concomitant blunting of microvilli. Electron micrographs from 42 couplets (from 8 separate preparations) with a canalicular space diameter of $2-4 \mu \mathrm{m}$ obtained $4 \mathrm{~h}$ after culture were compared with similar thin-section electron micrographs of 36 couplets ( 5 experiments) obtained immediately after isolation.

Table I gives the absolute values and ratios of the two morphometrically measured parameters obtained from freshly isolated couplets and those cultured for $4 \mathrm{~h}$ in L-15 media. Canalicular spaces in the 4-8-h sample were divided into two groups based on their ultrastructural appearance: one group with essentially normal microvilli and pericanalicular mor- 
Table I. Morphometric Analysis of the Effects of Time in Culture on Canalicular Membrane Perimeter (Pm) and Cross-sectional Area with and without Microvilli $( \pm M V)$ in IRHC

\begin{tabular}{|c|c|c|c|c|c|c|c|c|c|}
\hline \multicolumn{6}{|c|}{ Canalicular perimeter $(\mu \mathrm{m})$} & \multicolumn{4}{|c|}{ Cross-sectional area of canalicular lumen $\left(\mu \mathrm{m}^{2}\right)$} \\
\hline $\begin{array}{l}\text { Culture } \\
\text { time }\end{array}$ & $\begin{array}{c}\text { Canal } \\
\text { diameter }\end{array}$ & $\begin{array}{c}\text { Canal Pm } \\
+\mathrm{MV} \\
\mathrm{A}\end{array}$ & $\begin{array}{c}\text { Canal Pm } \\
-\mathbf{M V} \\
\text { B }\end{array}$ & $\begin{array}{c}\text { MV Pm } \\
\text { A - B }\end{array}$ & $\begin{array}{l}\text { Ratio } \\
\text { A/B }\end{array}$ & $\begin{array}{c}\text { Area }+M V \\
A\end{array}$ & $\begin{array}{c}\text { Area -MV } \\
\text { B }\end{array}$ & $\begin{array}{c}\text { MV area } \\
\text { A - B }\end{array}$ & $\begin{array}{l}\text { Ratio } \\
\text { A/B }\end{array}$ \\
\hline $\begin{array}{l}0-15 \mathrm{~min} \\
n=36\end{array}$ & $0-1 \mu \mathrm{m}$ & $10.8 \pm 5.4^{*}$ & $5.4 \pm 3$ & $4.8 \pm 2.6^{*}$ & $1.9 \pm 0.25^{\ddagger}$ & $1.3 \pm 1.14^{*}$ & $1.05 \pm 1.0$ & $0.25 \pm 0.16^{*}$ & $1.2 \pm 0.8$ \\
\hline \multicolumn{2}{|c|}{ Normal $(n=37)$} & $41.1 \pm 15^{*}$ & $23.0 \pm 6.8$ & $18.2 \pm 8.9^{*}$ & $1.8 \pm 0.3^{\ddagger}$ & $17.8 \pm 6.5^{*}$ & $16.9 \pm 6.3$ & $0.86 \pm 0.5^{*}$ & $1.05 \pm 0.03$ \\
\hline $\begin{array}{c}4-8 \mathrm{~h} \\
n=42\end{array}$ & $2-4 \mu \mathrm{m}$ & & & & & & & & \\
\hline \multicolumn{2}{|c|}{ Cholestatic $(n=5)$} & $25 \pm 7.1$ & $19.3 \pm 4.8$ & $5.4 \pm 3.6$ & $1.3 \pm 0.18$ & $13.7 \pm 3.6$ & $13.4 \pm 3.5$ & $0.38 \pm 0.31$ & $1.02 \pm 0.02$ \\
\hline
\end{tabular}

Hepatocyte couplets were analyzed immediately after isolation $(n=36)$ or after $4-8 \mathrm{~h}$ in culture $(n=42)$. Measurements were limited to those couplets whose lumens had expanded to a diameter of 2-4 $\mu \mathrm{m}$. Cholestatic couplets were defined as having widened pericanalicular ectoplasm and loss of microvilli. ${ }^{*} P<0.005$. ${ }^{\ddagger}$ Nonsignificant difference: normal couplets at 4-8 h compared with couplets immediately after isolation.

phology, and the other, cholestatic, group defined by thickening of the pericanalicular ectoplasm and loss of microvilli on the canalicular membrane, an appearance consistent with the characteristic ultrastructural morphology of cholestasis (10).

The actual area of the canalicular space after $4 \mathrm{~h}$ in culture measured from the electron micrographs in analysis $(A)$ was 95-97\% of the cross-sectional area measured when the microvilli were excluded in analysis (B). This latter measurement corresponded to the optical planimetry method. Since volume was calculated from the sum of all cross-sectional areas through the depth of the canalicular vacuole, the optical planimetry volume measurements should closely approximate the actual volume $(0.95: 1)$ since there was an error of overestimation of only $3-5 \%$ based on the electron micrograph studies. Furthermore, the error remained relatively constant when canalicular spaces of various diameters from 1 to $4 \mu \mathrm{m}$ were examined. Thus, the canalicular microvilli did not introduce a significant error in volume measurements as calculated by optical planimetry.
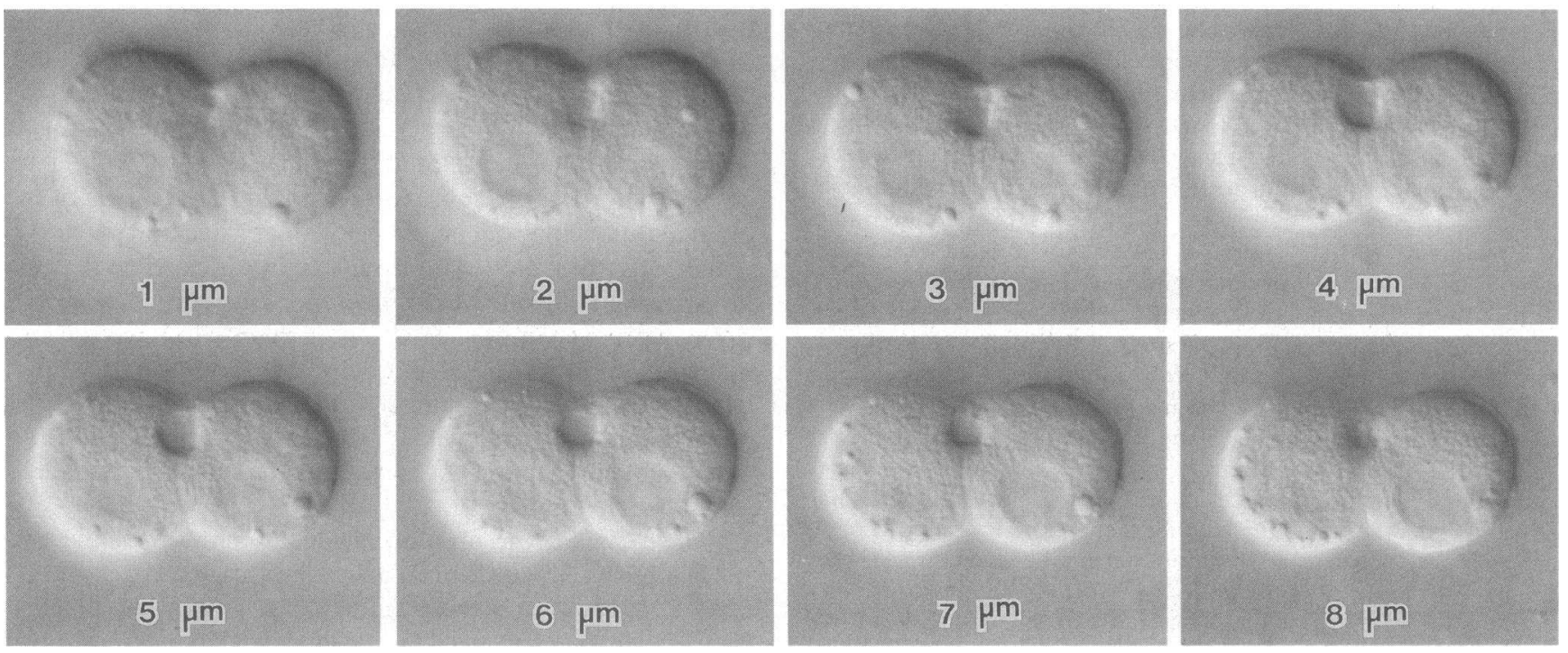

Figure 3. Optical planimetry of a single couplet. Cross-sectional images at $1-\mu \mathrm{m}$ intervals through the depth of a canaliculus from the top pole to the bottom end. (Nomarski optics, Kodak Panatomic-X, ASA32 film). 


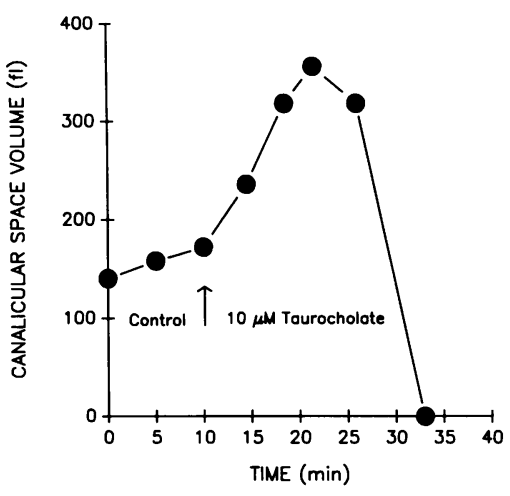

Figure 4. Effect of 10 $\mu \mathrm{M}$ taurocholate on rate of change of canalicular volume in femtoliters in isolated couplets. After several volume measurements by optical planimetry, the L-15 perfusion medium is changed to one containing $10 \mu \mathrm{M}$ taurocholate. Volume measurements are continued at 3-5-min intervals to determine rate of change of canalicular volume.

top of the canaliculus and proceeding through the middle of the space to the opposite end. These photographs demonstrate the moderately irregular shape and cross-sectional area of the canaliculus although there is a substantial loss of contrast compared with the video-enhanced microscopy from which actual measurements were made.

The shape of the canalicular space was only infrequently spherical or ovoid and was usually asymmetrical with the length of the canaliculus aligned variably, parallel or perpendicular to the z-axis (vertical axis) or at varying angles to it. Furthermore, expansion did not occur symmetrically in all dimensions. Thus, although calculations of canalicular volume extrapolated mathematically from two-dimensional measurements at the largest diameter and based on the uniform assumption of a spherical canalicular shape are simpler and less time consuming, they are not applicable to the large proportion of IRHC. Canalicular volume calculations, based on the above assumption, differ from planimetric measurements by $13 \pm 33 \%$, ranging from $-40 \%$ to $+114 \%$. Canalicular volumes were underestimated in $38 \%$ of couplets and overestimated in $62 \%$ of IRHC. Values of canalicular volume agreed to within $10 \%$ of planimetric volumes in $28 \%$ of couplets and to within $5 \%$ in only $14 \%$ of IRHC.

We next studied the basal secretion rate as calculated from the rate of canalicular volume change and compared this with the effect of superfusion of $10 \mu \mathrm{M}$ taurocholate (Fig. 4). Several measurements of the expanding canalicular volume were made in L-15 medium and represented the basal flow rate.

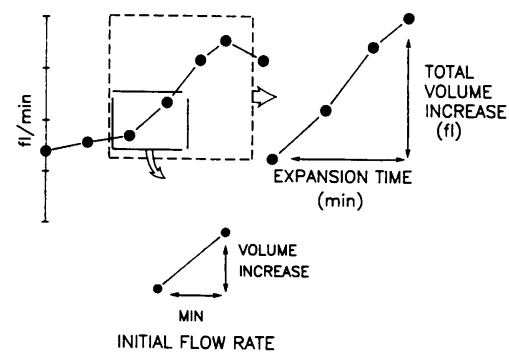

Figure 5. Calculation of measured parameters. Initial flow rate (in femtoliters/minute) is the increase in canalicular volume in femtoliters divided by the time in minutes of the duration of first period (3-5 min) of quantitation, in both the control period and after exposure to the bile acid. Total volume increase (in femtoliters) is the total secretion from the point of exposure to the bile acid to the point when the space begins to collapse, and expansion time (minutes) is the time elapsed between these two points.

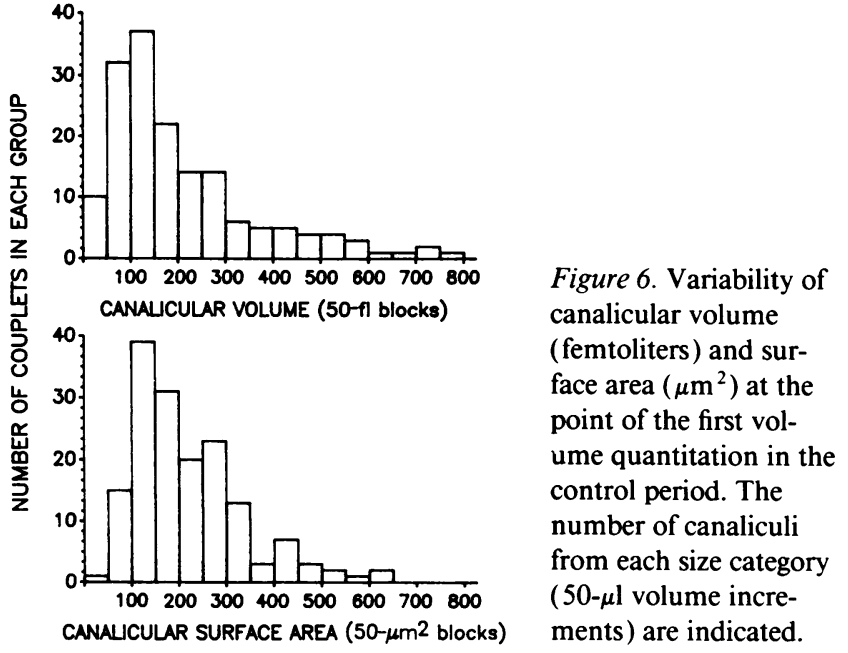

Taurocholate $(10 \mu \mathrm{m})$-containing L-15 medium was then superfused and provoked a canalicular space expansion that increased progressively over $20 \mathrm{~min}$ until the canalicular space either collapsed because of leakiness of the junctions or contracted. The secretory cycle was quantitated as illustrated (Fig. 5).

Fig. 6 represents the number and range of canalicular volumes and canalicular surface areas measured by this technique from a total of 161 couplets. A considerable variation is noted in the volumes and surface areas of the canalicular spaces observed during the 4-8 $\mathrm{h}$ after IRHC isolation.

Pooled data from a number of experiments is given in Table II. Basal bile flow rates averaged $3.8 \pm 1.3 \mathrm{fl} / \mathrm{min}$ in the couplet system in unmodified L-15 medium. L-15 medium is a defined medium containing amino acids and nutrients but is nominally $\mathrm{HCO}_{3}$-free and contains no specific growth factors. Taurocholate and ursodeoxycholate in $10-\mu \mathrm{M}$ concentrations both stimulated initial flow rate significantly and to comparable levels. Total volume also increased to comparable levels before leakiness or contractions occurred. The expansion cycle averaged $12 \pm 5 \mathrm{~min}$ after taurocholate administration and $18 \pm 9$ min after ursodeoxycholate, and correlated inversely with the canalicular volume at the point of stimulation with the bile acid ( $r=0.54, P<0.01$; Fig. 7$)$. Significant variability was noted in the initial flow rate in each treatment group despite the same stimulating dose of bile acid $(10 \mu \mathrm{M})$. This variation in secretory rates correlated linearly with the initial canalicular membrane surface area as illustrated in Fig. 8, sug-

Table II. Choleresis Induced by Bile Salts in IRHC

\begin{tabular}{cccc}
\hline & Initial flow rate & Total secretion & Expansion time \\
\hline & $(f l / \mathrm{min})$ & $(f)$ & $($ min $)$ \\
Control $(n=28)$ & $\begin{array}{c}3.8 \pm 1.3 \\
(\text { range } 1.8-5.5)\end{array}$ & & \\
$\begin{array}{c}\text { Taurocholate } \\
(10 \mu \mathrm{M}, n=40)\end{array}$ & $\begin{array}{c}14 \pm 7 \\
\text { (range 3-29) } \\
14 \pm 7\end{array}$ & $119 \pm 63$ & $12 \pm 5$ \\
$\begin{array}{r}\text { Ursodeoxycholate } \\
(10 \mu \mathrm{M}, n=24)\end{array}$ & $\begin{array}{c}137 \pm 74 \\
(\text { range 6-25) }\end{array}$ & & $18 \pm 9$ \\
\hline
\end{tabular}




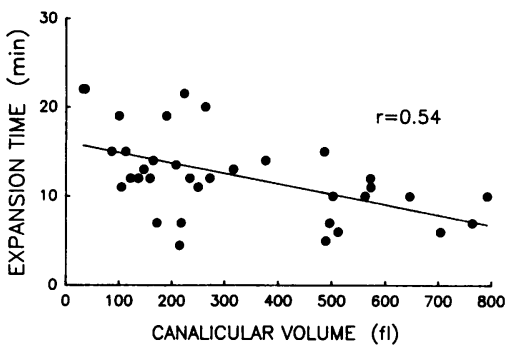

gesting that fluid flow in the couplet system is a function of the canalicular surface area and is presumably related to either the number of bile acid transporters on the canalicular membrane, its total hydraulic conductivity, or both. Similar correlations between flow rate and canalicular surface area were seen after ursodeoxycholic acid-induced secretion.

Fig. 9 shows the effect of various concentrations of ursotaurocholate on the bile flow rate in IRHC perfused with a KRB. The basal flow rate $(7.6 \pm 2.1 \mathrm{fl} / \mathrm{min}$ representing a $3.85 \pm 1.4 \%$ increase in canalicular vol $/ \mathrm{min}, n=19$ ) was significantly higher ( $\sim$ twofold $)$ than the basal flow rate in the L-15 medium ( see Table II ). $100 \mu \mathrm{M}$ taurocholate induced a significantly greater initial rate of secretion $(24.2 \pm 11.9 \mathrm{fl} / \mathrm{min}$ that represents a $12.7 \pm 8.3 \%$ increase in canalicular $\mathrm{vol} / \mathrm{min}$ after exposure to the bile acid, $n=25$ ) than $10 \mu \mathrm{M}$ taurocholate (16.6 $\pm 7.2 \mathrm{fl} / \mathrm{min}$, representing an $8.1 \pm 3.1 \%$ increase in canalicular vol $/ \mathrm{min}, n=14)$. Neither $100 \mu \mathrm{M}$ ursodeoxycholate $(17.2 \pm 7.2 \mathrm{fl} / \mathrm{min} ; \mathrm{a} 9 \pm 3.2 \%$ volume increase $/ \mathrm{min} ; n=10)$ nor $200 \mu \mathrm{M}$ ursodeoxycholate $(15.3 \pm 8.1 \mathrm{fl} / \mathrm{min}$, an $11.8 \pm 6 \%$ volume increase $/ \mathrm{min}, n=15$ ) induced a hypercholeresis compared with taurocholate. In taurine-depleted IRHC perfused with a $\mathrm{KRB}-\mathrm{HCO}_{3}$ buffer, no hypercholeresis was noted in the $100-\mu \mathrm{M}$ urso experimental group $(18.7 \pm 7 \mathrm{fl} / \mathrm{min}, n$ $=18)$ compared with $20 \mu \mathrm{M}$ taurocholate $(20 \pm 10 \mathrm{fl} / \mathrm{min}, n$ =11), similar to findings in normal IRHC.

Fig. 10 shows the effect of ursodeoxycholate on bile secretory rates in $\beta$-alanine-pretreated, taurine-depleted IRHC that were both grown and perfused in bicarbonate and growth factor containing nutrient medium (MEM supplemented with $10 \%$ FCS). This protocol also eliminated a transfer step from the L-15 growth medium to a salt solution (KRB perfusion buffer). Bile secretory rates were measured in IRHC exposed to $100 \mu \mathrm{M}$ concentrations of ursodeoxycholate and taurocholate and in untreated controls. Although the basal flow rates were similar in both groups (controls: $3.8 \pm 4.7 \mathrm{fl} / \mathrm{min}, n=18$; taurocholate: $4.8 \pm 4.2 \mathrm{fl} / \mathrm{min}, n=44$; and ursodeoxycholate: $5.1 \pm 3.4 \mathrm{fl} / \mathrm{min}, n=52), 100 \mu \mathrm{M}$ taurocholate induced a sig-

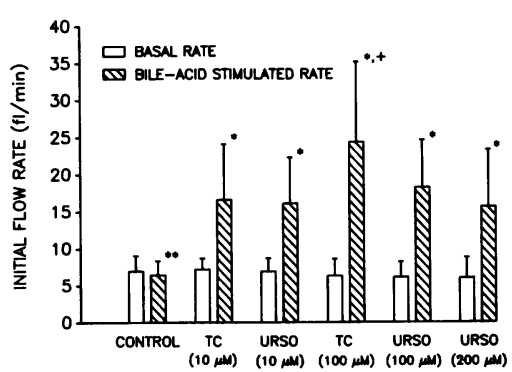

Figure 9. Effect of various concentrations of taurocholate and ursodeoxycholate on the bile secretory rate in IRHC perfused with KRB. ${ }^{*} P<0.01$ vs. paired basal secretory rates. ${ }^{+} P<0.05$ vs. 10 $\mu \mathrm{M}$ ursodeoxycholate and taurocholate and 100 and $200 \mu \mathrm{M}$ ursodeoxycholate. ${ }^{* *} \mathrm{NS}$ vs. paired basal rates.

nificantly greater maximal rate of choleresis than ursodeoxycholate (taurocholate: $16.8 \pm 11.4 \mathrm{fl} / \mathrm{min}, n=44$; ursodeoxycholate: $9 \pm 6.33 \mathrm{fl} / \mathrm{min}, n=52, P<0.01$ ), while the bile secretory rate in controls was significantly lower than either bile acid $(6.3 \pm 3.0 ; P<0.005)$.

These studies suggest that ursodeoxycholate-induced hypercholeresis in the intact liver does not occur by a direct acute effect of the bile acid on single hepatocyte secretory units. Norursodeoxycholic acid (50-100 $\mu \mathrm{M}$ ), kindly provided by Dr. Alan Hoffman, University of California San Diego Medical School (San Diego, CA), which is a C-23 bile acid that produces a hypercholeresis in vivo and is not conjugated to taurine/glycine significantly (26), also failed to induce a hypercholeresis in these IRHC (unpublished observations).

\section{Discussion}

IRHC in short-term culture provide a unique model that functions as a primary unit of canalicular secretion. The volume of the canalicular space increases as secretion occurs exclusively across the canalicular membrane independently of hormonal or blood-flow effects or distal modifications of secretion by bile ductular cells. The rate of volume increase in unmodified L-15 medium reflects the baseline secretion rate and is significantly enhanced after exposure to the choleretic bile acids, taurocholate and ursodeoxycholate. Thus, the IRHC model provides a novel opportunity to measure canalicular secretion using quantitative video-microscopy techniques that record changes in canalicular volume.

Techniques for volume measurement of cells and interspaces by microscopic optical planimetry have been previously

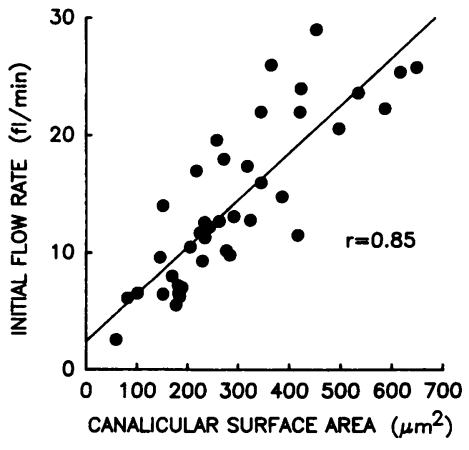

Figure 8. Correlation between the initial flow rate and the initial canalicular surface area, i.e., surface area of the canalicular space at the point of first exposure to the bile acid ( $P$ $<0.005$ ). Each point represents one experiment where the canalicular secretion rate is measured in a separate couplet.

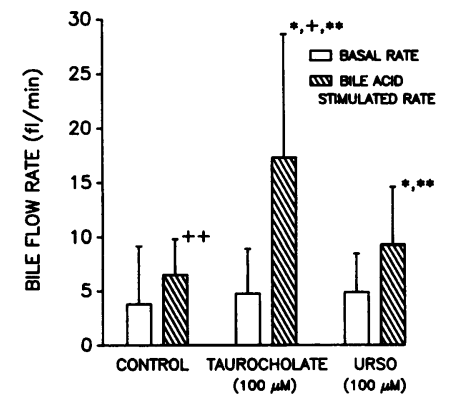

Figure 10. Effect of $100 \mu \mathrm{M}$ ursodeoxycholate and taurocholate compared with untreated controls on bile secretory rates in taurine-depleted IRHC, cultured and studied in bicarbonate-containing nutrient medium (MEM $+10 \%$ FCS). ${ }^{*} P<0.001$ vs. paired basal secretory rates; ${ }^{* *} P$ $<0.005$ vs. maximal secretory rate in untreated controls; ${ }^{+} P$ $<0.01$, taurocholate vs. ursodeoxycholate; ${ }^{++}$NS vs. paired basal control rates. 
validated by Spring et al. (17-19) and other investigators (30, 31 ). The canalicular space is frequently irregular in shape and does not expand symmetrically in all dimensions. Extrapolations of canalicular volume measurements from single measurements at the largest diameter result in significant under- or overestimations of canalicular volume and volume changes in the basal or stimulated state, and do not compensate for spontaneous modulation of canalicular shape with time. Nomarski (DIC) optics, which provide an extremely shallow depth focus, are ideal for optical sectioning of an irregular three-dimensional structure to quantitate its volume more accurately.

Video-microscopy also provides major advantages over visualization by the human eye or by cine photomicrography. A video imaging device reacts linearly to light intensity and thus can distinguish a larger number of gray scales in an image compared with the human eye which reacts logarithmically to light intensity and also accommodates automatically (32). It is possible to increase the numerical aperture of the condenser lens close to that of the objective lens, thereby increasing resolution while contrast in images is enhanced by adjusting the black level. Finally, data storage and quantitative analyses are easier to process compared with data on photographic film.

At the level of resolution of the light microscope, microvilli lining the canalicular membrane can not be distinguished. However, these structures (0.1-0.2 $\mu$ m diameter) account for a minor and relatively constant percentage of the total volume of the canaliculus and this percentage remains virtually constant as canalicular space size increases with time in culture. Thus, the space occupied by the microvilli represents an insignificant error in the volume measurements of the canaliculus.

As the canalicular space expands over $4 \mathrm{~h}$ in culture after plating, the total surface area of apical membranes increases without significant loss or flattening of microvilli. Therefore, the volume increase during this time cannot be explained by simple stretching of the membrane but must occur because of addition of new canalicular membrane with preservation of relatively normal microvillar architecture as the couplet reorganizes its remaining apical domain. The accrual of additional canalicular membrane presumably occurs incrementally with time over 4-8 h in culture over repeated expansion/collapse cycles. Previous studies from our laboratory suggest that this membrane reorganization process may depend on intact microfilament function since cytochalasin D prevents relocation of canalicular remnant $\mathrm{Mg}^{++} \mathrm{ATPase}$ to the remaining canalicular domain between the two hepatocytes (10). This IRHC model should, therefore, be of interest in studies of membrane domain targeting and reorganization.

Canalicular volume increases slowly $(3.8 \pm 1.3 \mathrm{fl} / \mathrm{min})$ in the basal L-15 medium and presumably represents the rate of nonbile acid-dependent canalicular bile formation in the isolated hepatocyte couplets in the absence of bile acids, serum, hormones, bicarbonate, and growth factors. The measured value represents $10-15 \%$ of the total basal rate of secretion (both bile acid-dependent and independent) calculated for two hepatocytes in the intact, bile duct-cannulated rat with a $10-\mathrm{g}$ liver containing $1 \times 10^{9}$ hepatocytes and $20-35 \%$ of the rate of basal secretion in the isolated perfused rat liver system (10-g liver, bile flow rate $1 \mu \mathrm{l} / \mathrm{min}$ per $\mathrm{g}$ liver wt without bile acid perfusion). Furthermore, when the perfusion medium contained bicarbonate, the basal rate of secretion increased by a factor of $\sim 2$ representing $40-70 \%$ of the basal secretory rate. However, additional systematic studies comparing bicarbon- ate-containing or bicarbonate-free perfusion media are needed to further study the role of bicarbonate in basal canalicular bile formation.

Secretion in the couplet system occurs into a closed space that is separated from the perfusion medium by tight junctional structures that display selective permeability and electrical resistances consistent with conductances across a leaky epithelium (12). Thus, hydrostatic pressure presumably builds up within the lumen as the space distends and the canalicular membrane is stretched in a cycle of expansion and after addition of choleretic bile acids. This pressure may also result in lower basal secretory rate observed. To minimize the effects of hydrostatic forces on bile acid-stimulated secretion, initial flow rates were used in these studies to compare the choleretic effects of sodium taurocholate and ursodeoxycholate.

Additions of either taurocholate and ursodeoxycholate (10 $\mu \mathrm{M})$ resulted in stimulation of secretion to comparable levels (initial flow rate, $14 \pm 7 \mathrm{fl} / \mathrm{min}$ each). This bile acid-induced increment in the initial flow rate approximated $50-100 \%$ of that expected for two hepatocytes in the intact bile-duct cannulated rat (10-g liver) for $10-\mu \mathrm{M}$ concentrations of taurocholate. Thus, with appropriate controls the IRHC is a suitable model for comparing both the effects of choleretic agents on stimulated bile secretory rates and for the role of various factors on basal bile secretion.

The initial rate of expansion after bile acid stimulation was quite variable and this may limit rapid detection of agents that are weakly choleretic at the canalicular level. While some of the variability in secretory rates might result from differences in the zonal origin of the couplets (centrizonal or periportal), the initial flow rates correlated well with the surface area of the canaliculus at the time of addition of the bile acids, suggesting that the rate of canalicular fluid secretion is either a function of the number of transport carriers on the canalicular membrane or a larger net hydraulic conductivity for $\mathrm{H}_{2} \mathrm{O}$. Since it is likely that during acute bile acid choleresis the relatively rapid expansion of the canalicular space is the result of canalicular stretching rather than significant addition of new membrane, another physical factor that could account for this phenomenon is described by Laplace's law, which predicts that less force (secretion pressure) is required to overcome the wall tension in the larger canalicular spaces. However, Laplace's law would apply more closely when the canalicular space is maximally distended immediately before the time when the space contracts or collapses rather than during the earliest phase of expansion, i.e., when the initial flow rate is calculated.

In the present study the duration of the expansion time, i.e., from the point of stimulation with the bile acid to the onset of collapse, was also variable but was inversely related to the degree of "filling" of the canalicular space at the point of stimulation with the choleretic agent. Thus, canalicular spaces that were expanded relative to their surface area (initial canalicular volume/canalicular surface area ratio $>1$ ) contracted or collapsed sooner than those in which volume was relatively less compared with their surface area. This inverse relationship between the degree of expansion and time of collapse strongly suggests that the collapse/contraction phenomenon is not spontaneous but is a functiol. of the filling pressure and distension of this closed canalicular space.

When the canalicular space is maximally expanded, leaks develop in the tight junction and the space collapses. However, this collapse may subsequently also involve active contraction 
of the cytoskeleton in the submembrane region since microfilament inhibitors (cytochalasins) inhibit this contraction process (33). After collapse or contraction of the space the tight junctions apparently reseal and another cycle of expansion begins, according to Phillips' group. Rapid reassembly and repair of transiently ruptured tight junctions has been observed previously in organ and tissue culture systems $(34,35)$. The frequency of collapse/contraction episodes in our study was much slower than the 3-5-min frequency reported previously by Phillips and co-workers (36). This difference may be accounted for in part by a lower basal secretion rate in the unmodified and unenriched L-15 medium. However, the phase-contrast optics imaging system, as used by these authors in a single plane of focus at the largest diameter, may, during time-lapse cine-micro-photography, register a modulation of canalicular shape as a partial contraction and thereby record a higher rate of contractions (37).

A major finding in these studies is the absence of hypercholeresis after exposure of IRHC to various concentrations of ursodeoxycholate $(10,100$, or $200 \mu \mathrm{M})$ compared with taurocholic acid, suggesting that the ursodeoxycholate-induced hypercholeresis observed in the intact liver does not occur by a direct acute effect of this bile acid on an individual hepatocyte secretory unit. This conclusion is strengthened by the absence of hypercholeresis in isolated couplets that were depleted of taurine. This protocol minimizes taurine conjugation of ursodeoxycholate to tauroursodeoxycholate which is not hypercholeretic and might otherwise mask an acute hypercholeretic effect. Hypercholeresis was absent in couplets perfused with either KRB or a bicarbonate-containing nutrient medium, MEM. Preliminary experiments in collaboration with Dr. A. Hoffman (University of California, San Diego) revealed that norursodeoxycholate, a C-23 bile acid that is not significantly amidated and is excreted largely unconjugated into bile (26), was also not hypercholeretic in this IRHC system.

It is possible that an ursodeoxycholic acid-induced hypercholeresis is masked in the IRHC model due to increased tight junctional permeability to putative osmotically active inorganic ionic species that might be secreted in response to this bile acid. However, preliminary studies demonstrate that cAMP analogues and forskolin, a direct activator of endogenous adenylate cyclase, which induce secretion in a variety of epithelia primarily by stimulating electrolyte secretion, also stimulate canalicular bile flow in $\operatorname{IRHC}(38,39)$.

Thus, ursodeoxycholate-induced hypersecretion in the intact animal could either be based on repeated cycling of the bile acid across the canalicular membrane (the cholehepatic shunt pathway, 25) or result from a primary action of the bile acid at sites distal to the bile canaliculus.

Another interesting observation is the lack of difference in choleretic response between $10-, 100-$, or $200-\mu \mathrm{M}$ concentrations of ursodeoxycholate in contrast to significant differences in bile secretion induced by $10-$ and $100-\mu \mathrm{M}$ taurocholate. The initial flow rates measured in these studies reflect the osmotic effects of bile acids secreted into the canalicular lumen. These observations suggest that saturation of the bile acid transport mechanisms occurs at lower intracellular concentrations of ursodeoxycholic acid than taurocholic acid in this primary secretory unit. Since ursodeoxycholic acid produces a dosedependent increase in bile flow in vivo, recruitment of additional secretory units within the hepatic lobule may occur rather than increased secretion at the level of each secretory unit. If this hypothesis is true, relatively more secretory units should be recruited within the hepatic lobule by ursodeoxycholate compared with equivalent doses of taurocholate.

\section{Acknowledgments}

This work was supported in part by a Pilot Project from a National Institutes of Health Liver Center grant DK-34984 and DK-25636. Dr. Strazzabosco was a recipient of North Atlantic Treaty OrganizationConsiglio Nazionale delle Ricerche Advanced Fellowship Program Award.

\section{References}

1. Boyer, J. L. 1986. Mechanisms of bile secretion and hepatic transport. In Physiology of Membrane Disorders. T. E. Andriole, F. Hoffman, and D. D. Fanestil, editors. Plenum Publishing Corp., New York. 609-636.

2. Klassen, C. D., and J. B. Watkins. 1984. Mechanisms of bile formation, hepatic uptake and biliary excretion. Pharmacol. Rev. 36:1-67.

3. Scharschmidt, B. F., and R. W. van Dyke. 1983. Mechanisms of hepatic electrolyte transport. Gastroenterology. 85:1199-1214.

4. Graf, J. 1983. Canalicular bile salt-independent bile formation: concepts and clues from electrolyte transport in rat liver. Am. J. Physiol. 244:G233-G246.

5. Arias, I. M. 1986. Mechanisms and consequences of ion transport in the liver. In Progress in Liver Diseases. Vol. VIII. H. Popper and F. Schaffner, Editors. Grune \& Stratton, Inc., New York. 145-160.

6. Inoue, M., R. Kinne, T. Tran, and I. M. Arias. 1982. Taurocholate transport by rat liver sinusoidal membrane vesicles: evidence of sodium cotransport. Hepatology (Baltimore). 2:572-579.

7. Meier, P. J., E. S. Sztul, A. Reuben, and J. L. Boyer. 1984. Structural and functional polarity of canalicular and basolateral plasma membrane vesicles isolated in high yield from rat liver. J. Cell Biol. 98:991-1000.

8. Inoue, M., R. Kinne, T. Tran, L. Biempica, and I. M. Arias. 1983. Rat liver canalicular vesicles: isolation and topographical characterization. J. Biol. Chem. 258:5183-5188.

9. Graf, J., A. Gautam, and J. L. Boyer. 1984. Isolated rat hepatocyte couplets: a primary secretory unit for electrophysiological studies of bile secretory function. Proc. Natl. Acad. Sci. USA. 81:6516-6520.

10. Gautam, A., O. C. Ng, and J. L. Boyer. 1987. Isolated rat hepatocyte couplets in short-term culture: structural characteristics and plasma membrane reorganization. Hepatology (Baltimore). $7(2): 216-223$.

11. Oshio, C., and M. J. Phillips. 1981. Contractility of bile canaliculi: implications for liver function. Science (Wash. DC). 212:10411042.

12. Graf, J., R. M. Henderson, B. Krumpholz, and J. L. Boyer. 1987. Cell membrane and transepithelial voltages and resistances in isolated rat hepatocyte couplets. J. Membr. Biol. 95:241-254.

13. Henderson, R. M., J. Graf, and J. L. Boyer. 1987. Na-H exchange regulates intracellular $\mathrm{pH}$ in isolated rat hepatocyte couplets. Am. J. Physiol. 252:G109-G113.

14. Boyer, J. L., O. C. Ng, and A. Gautam. 1985. Formation of canalicular spaces in isolated hepatocyte couplets. Prog. Am. Assoc. Physicians. XCVIII:21-29.

15. Sakisaka, S., O. C. Ng, J. Bouderdaben, and J. L. Boyer. 1988. Evidence for tubulovesicular transcellular transport of horseradish peroxidase (HRP) in isolated rat hepatocyte couplets in short-term culture. Gastroenterology. 95:793-804.

16. Boyer, J. L., A. Gautam, and J. Graf. 1988. Mechanisms of bile secretion: insights from the hepatocyte couplet. Semin. Liver Dis. 8:308-316.

17. Spring, K. R., and A. Hope. 1979. Fluid transport and dimensions of cells and interspaces of living Nectarus gallbladder. J. Gen. Physiol. 73:287-305. 
18. Persson, B.-E. and K. R. Spring. 1982. Gallbladder epithelia cell hydraulic water permeability and volume regulation. J. Gen. Physiol. 79:481-505.

19. Fisher, R. S., and K. R. Spring. 1984. Intracellular activities during volume regulation by Nectarus gallbladder. J. Membr. Biol. 78:187-199.

20. Gautam, A., D. M. Scaramuzza, and J. L. Boyer. 1986. Quantitative assessment of primary canalicular secretion in isolated rat hepatocyte couplets by optical planimetry. Gastroenterology. 90(5):1727a. (Abstr.)

21. Dumont, M., S. Erlinger, and S. Uchman. 1980. Hypercholeresis induced by ursodeoxycholic acid and 7-ketolithocholic acid in the rat: possible role of bicarbonate transport. Gastroenterology. 79:82-89.

22. Lake, J. R., R. W. Van Dyke, and B. F. Scharschmidt. 1987. Effect of $\mathrm{Na}^{+}$replacement and amiloride on ursodeoxycholic acidstimulated choleresis and biliary bicarbonate secretion. Am. J. Physiol. 252 (Gastrointest. Liver Physiol. 15):G163-G169.

23. Renner, E. L., J. R. Lake, E. J. Cragoe, R. W. Van Dyke, and B. F. Scharschmidt. 1988. Ursodeoxycholic acid choleresis: relationship to biliary $\mathrm{HCO}_{3}^{-}$and effects of $\mathrm{Na}^{+}-\mathrm{H}^{+}$exchange inhibitors. $\mathrm{Am}$. J. Physiol. 254 (Gastrointest. Liver Physiol. 17):G232-G241.

24. Kitani, K., and S. Kanai. 1985. Ursodeoxycholate-induced choleresis in taurine-deprived and taurine-supplemented rats. Jpn. J. Physiol. 35:443-462.

25. Moseley, R. H., P. J. Meier, P. S. Aronson, and J. L. Boyer. 1986. Na-H exchange in rat liver basolateral but not canalicular membrane vesicles. Am. J. Physiol. 260:G35-43.

26. Yoon, Y. B., L. R. Hagey, A. F. Hoffman, D. Gurantz, E. L. Michelotti, and J. H. Steinbach. 1986. Effect of side-chain shortening on the physiologic properties of bile acids: hepatic transport and effect on biliary secretion of 23-Norursodeoxycholate in rodents. Gastroenterology. 90:837-852.

27. Blitzer, B. L., S. L. Ratoosh, C. B. Donovan, and J. L. Boyer. 1982. Effects of inhibitors of $\mathrm{Na}^{+}$-coupled ion transport on bile acid uptake by isolated rat hepatocytes. Am. J. Physiol. 243:G48-53.

28. Strange, K., and K. R. Spring. 1987. Absence of significant cellular dilution during ADH-stimulated water absorption. Science (Wash. DC). 235:1068-1070.

29. Shaffer, J. E., and J. J. Kocsis. 1981. Taurine mobilizing effect of beta alanine and other inhibitors of taurine transport. Life Sci. 28:2727-2736.

30. Capri-Medina, P., B. Lindemann, E. Gonzalez, and G. Whittembury. 1984. The continuous measurement of tubular volume changes in response to step changes in contraluminal osmolality. Pfluegers Arch. Eur. J. Physiol. 400:343-348.

31. Davis, C. W., and A. L. Finn. 1981. In Membrane Biophysics 1. Physical Methods in the Study of Epithelia. M. A. Dinno et al., editors. Alan R. Liss, Inc., New York. 25-36.

32. Inoue, S. 1986. Physiological characteristics of the eye. In Videomicroscopy. Plenum Publishing Corp. 71-92.

33. Phillips, M. J., C. Oshio, M. Miyairi, and C. R. Smith. 1983. Intrahepatic cholestasis as a canalicular motility disorder: evidence using cytochalasin. Lab Invest. 48:205-211.

34. Meldolesi, J., G. Castiglioni, R. Parma, N. Nassivera, and P. DeCamilli. 1978. $\mathrm{Ca}^{++}$-dependent disassembly and reassembly of occluding junctions in guinea pig pancreatic acinar cells. Effect of drugs. J. Cell Biol. 79:156-172.

35. Kachar, B., and P. DaSilva. 1981. Rapid massive assembly of tight junction strands. Science (Wash. DC). 213:541-544.

36. Miyairi, M., C. Oshio, S. Watanabe, C. R. Smith, I. M. Yousef, and M. J. Phillips. 1984. Taurocholate accelerates bile canalicular contractions in isolated rat hepatocytes. Gastroenterology. 87:788792.

37. Boyer, J. L. 1987. Contractile activity of bile canaliculi: contraction or collapse? Hepatology (Baltimore). 7(1):190-192.

38. Gautam, A., A. Ma, T. Mcguire, J. N. Forrest, E. R. Gordon, and J. L. Boyer. 1986. Forskolin, but not its cyclase inactive analog, stimulates choleresis in the isolated perfused rat liver. Hepatology (Baltimore). 1169a. (Abstr.)

39. Nathanson, M. J., and A. Gautam. 1988. Activation of endogenous adenylate cyclase ( $\mathrm{AC}$ ) stimulates choleresis in isolated rat hepatocyte couplets (IRHC). Gastroenterology. 94:619a. (Abstr.) 